\title{
ABSTRACTS
}

Abstracts on this page have been chosen and

edited by Dr Trevor Watts

ENDODONTICS; CARDIOLOGY

\section{Pulpal inflammation and incidence of coronary heart disease}

Joshipura KJ, Pitiphat W et al. J Endod 2006; 32: 99-103

In a group of health professionals, there was a small association between endodontic treatment (RCT) and subsequently diagnosed coronary heart disease.

There is a possible effect of inflammatory oral disease upon cardiovascular health, but current evidence is debatable. This study investigated data from 34,683 men included in a large US study from 1986 to 2000. RCT was used as a surrogate for pulpal inflammation, and 1275 cases of CHD were subsequently diagnosed in subjects free from it in 1986.

Analysis was performed with adjustment for age, smoking and family history of myocardial infarction. In subjects with 1 RCT between 1976 and 1996, the relative risk (RR) of CHD was 1.21 (CI 1.05-1.4), and for those with 2 RCTs, RR was 1.24 (1.04-1.47). RRs were not significant for RCT before 1976, or between 1987 and 1996, or for assessments of caries. In subgroups of dentists, younger subjects and current smokers, all with 2 or more RCTs between 1976 and 1996, RRs were moderately higher.

doi:10.1038/sj.bdj.4814045

\section{PERIODONTOLOGY; ORAL MEDICINE}

\section{Longitudinal evaluation of loss of} attachment in HIV-infected women compared to HIV-uninfected women

Alves M, Mulligan R et al. J Periodontol 2006; 77: 773-779

Rate of attachment loss was similar in both groups.

While certain types of periodontal disease have been associated with HIV infection and AIDS, there is little information on the progression of periodontal attachment loss in HIV infection. Furthermore, little is known about possible beneficial effects of HAART (highly-active anti-retroviral treatment) on periodontal health of HIV+ subjects. In this longitudinal study, $584 \mathrm{HIV+}$ and 151 HIV- women have been followed from 1995 in at least 2 separate visits.

Significantly more HIV+ subjects had used heroin, but significantly fewer used tobacco, marijuana or crack cocaine. HIV- subjects had higher educational attainments. There was no significant difference between groups in probing depth or attachment level change or tooth loss. There was marginally (but significantly) less pocket deepening in HIV + subjects who were on HAART than in those who were not, but no differences in loss of attachment or teeth.
ENDODONTICS; PHARMACOLOGY

\section{A Cochrane systematic review finds no evidence to support the use of antibiotics for pain relief in irreversible pulpitis}

Keenan JV, Farman AG et al. J Endod 2006; 32: 87-92

The limited available evidence suggests that antibiotics are inappropriate.

Irreversible pulpitis is appropriately treated by pulpectomy followed by root canal treatment, but some dentists prescribe antibiotics in the belief that this can stop the pain. This review sought randomised controlled trials (RCTs) of antibiotics versus placebo in adults with a single tooth diagnosed with irreversible pulpitis.

From an initial 35 potential papers, 31 were excluded as not meeting criteria. A further 2 studies were excluded because antibiotics were used adjunctively to endodontic treatment, and one more was a retrospective comparison. The remaining study was a double-blind RCT in 40 subjects, in which oral penicillin was the active agent, and analgesics were given for pain relief over a 7 day period. The RCT found no difference between groups in pain scores or amount of analgesics used; the authors judged it wellconstructed but underpowered, and recommended further trials.

doi:10.1038/sj.bdj.4814046

\section{MPLANT DENTISTRY; PROSTHODONTICS}

\section{Implant-retained mandibular} overdentures versus conventional dentures: 10 years of care and aftercare Visser A, Meijer JA et al. Int J Prosthodont 2006; 19: 271-278 Implant-supported overdentures required more time and treatment. Implant-supported overdentures reduce problems of lower denture retention and stability, but long-term follow-up studies on aftercare are lacking. In this Dutch study, patients referred because of repeated lower denture retention problems were randomised to 5 groups: overdenture (OD) on 2 implants $(n=30)$ or conventional denture (CD: 30 ), both with bone 8-15 mm high, OD on 2 implants (32) or CD after vestibuloplasty (30) or CD (29), all with bone 16-25 mm.

In the 5 respective groups, 10 yrs follow-up was completed by 29, 25, 30, 25 and 24 subjects. Mean treatment times in minutes for these groups were: surgery period, 150, 0, 166, 149, 0; surgical aftercare, including provision of implants in some CD subjects: $41,78,35$, 31,78 ; prosthetic care, 207, 151, 198, 155, 157; and prosthetic aftercare, 354, 250, 266, 238, 306. There were no significant differences in mean times for prosthetic care and aftercare even including those subjects with CDs who subsequently received ODs, but the initial OD groups took significantly more time for surgical care and aftercare. The authors note that patient satisfaction is greater with ODs.

doi:10.1038/sj.bdj.4814048 